\title{
Polar stratospheric cloud threshold temperatures in the 1995-1996 arctic vortex
}

\author{
James M. Rosen, ${ }^{1}$ Norman T. Kjome, ${ }^{1}$ Niels Larsen, ${ }^{2}$ Bjørn M. Knudsen, ${ }^{2}$ \\ Esko Kyrö, ${ }^{3}$ Rigel Kivi, ${ }^{3}$ Juha Karhu, ${ }^{3}$ Roland Neuber, ${ }^{4}$ and Ingo Beninga ${ }^{4}$
}

\begin{abstract}
Balloon-borne backscattersondes have been used to study the relationship between particle scattering and ambient temperature near the vertical edge of arctic polar stratospheric clouds (PSCs) as well as to delineate the cloud type occurrence probability as a function of temperature. The observed typical threshold temperatures as a function of altitude are about $1^{\circ} \mathrm{K}$ warmer than the temperature $T_{\mathrm{STS}}$ expected for rapid growth of supercooled ternary solution aerosols. A more descriptive analysis shows that the threshold temperatures occur over a definable range of temperatures and tend to cluster near, but somewhat warmer than, $T_{\mathrm{STS}}$. Considering the experimental and theoretical uncertainties, this difference may not be significant. The probability of type Ib PSC occurrence shows a dramatic increase at $T_{\text {STs }} \pm 1^{\circ} \mathrm{K}$, while for type Ia PSCs the probability is roughly constant at $10 \%$ for temperatures below the formation point of nitric acid trihydrate $\left(T_{\mathrm{NAT}}\right)$.
\end{abstract}

\section{Introduction}

In spite of diverse field observations, laboratory measurements, and new theoretical developments some important aspects of type I polar stratospheric cloud (PSC) formation mechanisms still remain unclear. Initially two classes of type I PSCs (Ia and Ib) were defined from the characteristic properties of lidar backscatter [Toon et al., 1990; Browell et al., 1990]: Ia particles are apparently larger, are of smaller concentration, have lower backscatter ratios, and are nonspherical as implied from the depolarization in the backscatter; Ib particles appear to be smaller, to be more numerous, to display higher backscatter ratios, and to be probably spherical or nearly spherical.

It was initially proposed that Ia PSCs would form by condensation on only the small fraction of particles present that could serve as active nuclei at the low supersaturations associated with a slowly cooling air mass [Toon et al., 1990]. Fast cooling would give rise to higher supersaturations, leading to condensation on a larger number or on all particles present, which, in turn, would result in the smaller particle size associated with Ib PSCs. However, it is now recognized that this simple explanation cannot be generally applicable since mesoscale temperature fluctuations (MTF) would effectively result in most air parcels experiencing high cooling rates [Murphy and Gary, 1995].

It is generally believed that type I PSCs are formed largely from nitric acid and water vapors available in the ambient atmosphere. Initially, it was suggested that type I PSCs were composed of nitric acid trihydrate (NAT), a solid crystal [Toon et al., 1986; Crutzen and Arnold, 1986]. This proposal was at

\footnotetext{
${ }^{1}$ Department of Physics and Astronomy, University of Wyoming, Laramie.

${ }^{2}$ Danish Meteorological Institute, Copenhagen, Denmark.

${ }^{3}$ Finnish Meteorological Institute, Sodankylä, Finland.

${ }^{4}$ Alfred Wegener Institute of Polar and Marine Research, Potsdam, Germany.

Copyright 1997 by the American Geophysical Union.

Paper number 97JD02701.

0148-0227/97/97JD-02701\$09.00
}

first supported by observations that PSCs contained nitric acid [Fahey et al., 1989] and by laboratory evidence indicating that NAT would form at the same general temperatures where PSCs were found [Hanson and Mauersberger, 1988]. However, more extensive observations and critical examinations indicated that PSCs were usually not present until the temperature dropped several degrees below that expected for the formation of NAT ( $\left.T_{\text {NAT }}\right)$. This apparent discrepancy could be understood if effective nuclei were unavailable for normal condensation and high supersaturations were necessary to force condensation on a few somehow special particles present. Such a scenario would be consistent with Ia PSC formation.

More recently, thermodynamic models of liquid supercooled ternary systems (STS) involving $\mathrm{H}_{2} \mathrm{O}, \mathrm{H}_{2} \mathrm{SO}_{4}$, and $\mathrm{HNO}_{3}$ have been proposed [Tabazadeh et al., 1994a, b; Carslaw et al., 1995; Luo et al., 1995; Taleb et al., 1996]. In general, these models predict a significant uptake of water and nitric acid vapors at a fairly well-defined temperature $\left(T_{\text {STS }}\right) \sim 4-5^{\circ} \mathrm{K}$ below $T_{\text {NAT }}$ and $\sim 2-3^{\circ} \mathrm{K}$ above the water ice formation temperature $T_{\text {ice }}$. Seemingly convincing, but select, observational evidence has been presented showing the formation of PSCs with Ib characteristics at a temperature consistent with $T_{\text {STs }}$ [Carslaw et al., 1994; Drdla et al., 1994; Dye et al., 1996; Beyerle et al., 1997]. However, the majority of these observations are not consistent with PSCs forming at $T_{\text {STS }}$ but at a somewhat warmer and broader range of temperatures [Tabazadeh and Toon, 1996].

Koop and Carslaw [1996] have described a multiple component system in which frozen and cooling background stratospheric aerosol particles can undergo a sudden melting and dramatic growth over only a $1^{\circ} \mathrm{K}$ temperature range at a welldefined temperature (deliquescent temperature $T_{d}$ ). As these authors indicate, such an effect should be observable in existing and future field measurements. This deliquescent effect should not be confused with the deliquescence of the liquid background sulfuric acid aerosol.

In this study we use a database of PSC observations obtained from balloon-borne instrumentation employing a backscattersonde to examine what we define as the threshold temperature at which PSCs are just detectable. In addition, a probability of 
occurrence for type Ia and Ib PSCs in relation to $T_{\mathrm{NAT}}$ and $T_{\mathrm{STS}}$ is also being developed from the basic field measurements.

\section{Instrumentation}

The field observations were made with a balloon-borne backscattersonde [Rosen and Kjome, 1991]. This instrument measures the local aerosol backscatter at 490 (B490) and 940 (B940), ozone concentration, pressure, temperature and tropospheric relative humidity, with a vertical resolution of about $30 \mathrm{~m}$. A notable and important feature of the technique is that the aerosol is sampled in its natural temperature and pressure environment, thus eliminating operational questions related to particle volatility effects. The final aerosol data products and profiles are very similar to those obtained by high-resolution, low-noise lidar systems. The unsmoothed background aerosol profiles in the $20 \mathrm{~km}$ altitude region indicate that the overall instrument point-to-point signal noise is of the order of $1 \%$ or less for the red backscatter channel. Thus small changes in aerosol can be reliably detected. Since the color index (CI) (defined as B940/B490) is size sensitive, some information concerning the general particle size can be deduced from the measurements. However, for small values of B490 the statistical fluctuations in the CI may become quite large, in which case it will be necessary to consider the characteristic value of several successive data points.

\section{Field Sites}

Data for this study were obtained from a total of 22 backscattersonde flights made during the 1995-1996 winter season from Thule, Greenland (Pituffik, $76.5^{\circ} \mathrm{N}, 68.8^{\circ} \mathrm{W}$ ), Scoresbysund, Greenland (Illoqqortoormiut, $70.5^{\circ} \mathrm{N}, 22.0^{\circ} \mathrm{W}$ ), Sondre Stromfjord (Kangerlussuaq, $67.0^{\circ} \mathrm{N}, 50.9^{\circ} \mathrm{W}$ ), Sodankylä, Finland $\left(67.4^{\circ} \mathrm{N}, 26.6^{\circ} \mathrm{E}\right)$, and $\mathrm{Ny}$ Alesund, Spitsbergen $\left(78.9^{\circ} \mathrm{N}\right.$, $11.9^{\circ} \mathrm{E}$ ). Experience indicates that stratospheric air over these stations, especially Sodankylä, is frequently under the influence of orographic forcing. Thus soundings at these locations may experience temperature extremes not apparent in synoptic data.

\section{Meteorological Setting}

Conditions characterizing the 1995-1996 winter polar vortex have been summarized by Manney et al. [1996], Santee et al. [1996], and Naujokat and Pawson [1996]. The stratosphere experienced colder temperatures than any of the previous 17 seasons with the nominal threshold for PSC formation being exceeded for more than 2.5 months. Temperatures reported by the radiosonde network were some of the lowest ever experienced. The vortex remained well developed and intact throughout the season until early to middle March, when the temperatures began to increase. Thus the 1995-1996 season provided an unusual opportunity for numerous type I and II PSC observations.

Another important aspect of the 1995-1996 vortex was the relatively small stratospheric aerosol background concentration. This condition made it possible to detect PSCs that perturbed the backscatter signal by only a few percent. Thus threshold PSCs could be detected with good sensitivity.

\section{Results and Analysis}

\subsection{Example Sounding}

Figure 1 illustrates the results of a winter arctic backscatter-

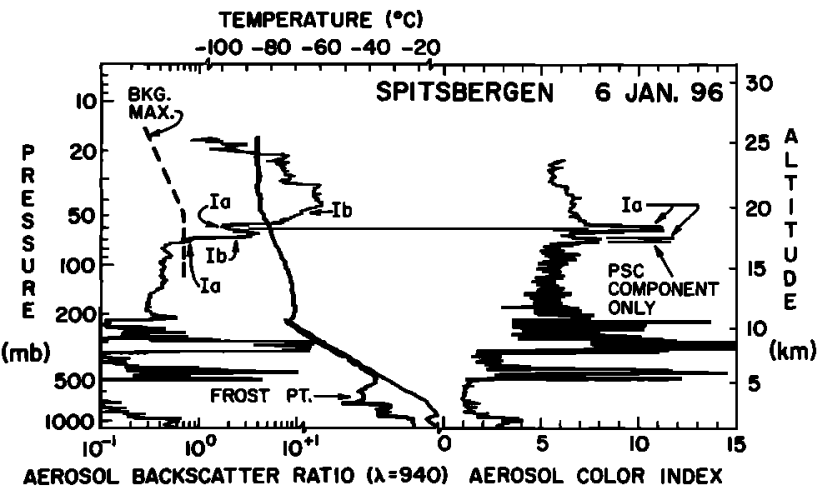

Figure 1. A backscattersonde sounding through a polar stratospheric cloud (PSC) over Spitsbergen. The dashed line associated with the aerosol backscatter ratio represents the background envelope maximum for non-PSC conditions. The lower temperature curve is the frost point as obtained with the Vaisala sensor. The higher value in the color index profile represents the color index of the aerosols in excess of the background aerosol, i.e., the PSCs exclusively. No data smoothing has been done in this illustration.

sonde flight with full resolution (no data smoothing). In this sounding nearly pure molecular scattering was observed in the dry air region (500-700 mbar) above the well-defined planetary boundary layer. Relatively weak clouds in the upper troposphere were observed concurrently with the high relative humidity implied by the frost point profile. It is interesting to note that the launch personnel specifically reported no visible clouds in the night sky. There was a very sharp division at the tropopause between the tropospheric and stratospheric air as indicated in the backscatter profile and ozone profile (ozone not shown). Background stratospheric aerosol was observed to about 75 mbar, where a small but significant increase was detected that can be associated with PSC activity and the more extended layer above. At the same altitude (the base of the main PSC layer) the aerosol color index increased significantly. As can be noted from the additional analysis given in the figure, the color index of the PSC component was considerably larger than that of the background aerosol, providing high definition of the layer base. Apparently, two thin layers of type Ia PSCs were present near the base of the main layer which, for the most part, was composed of type Ib PSCs. A more formal backscattersonde-based identification of type Ia and Ib PSCs is given below. A small temperature variation and structure in the ozone profile (not shown) suggests that these layers probably can be associated with filamentary-type transport similar to that described by Schoeberl and Newman [1995].

Figure 1 also illustrates the basic noise level of the measurements for the conditions encountered. In the background stratosphere the backscattersonde signal in the red channel displays minimal noise. Quantitatively, the standard deviation in the average of 10 consecutive data points for the total backscatter ratio (molecular plus aerosol) yields typical values of $\sim 1 \%$, while the corresponding value for just the aerosol component, which is the parameter used in Figure 1, is $\sim 4 \%$. The color index for background aerosols shows more noise because the blue channel has a correspondingly higher standard deviation in the aerosol component because of the fact that the total backscatter is close to the molecular backscatter. As can be seen in Figure 1, the color index noise is greatly 


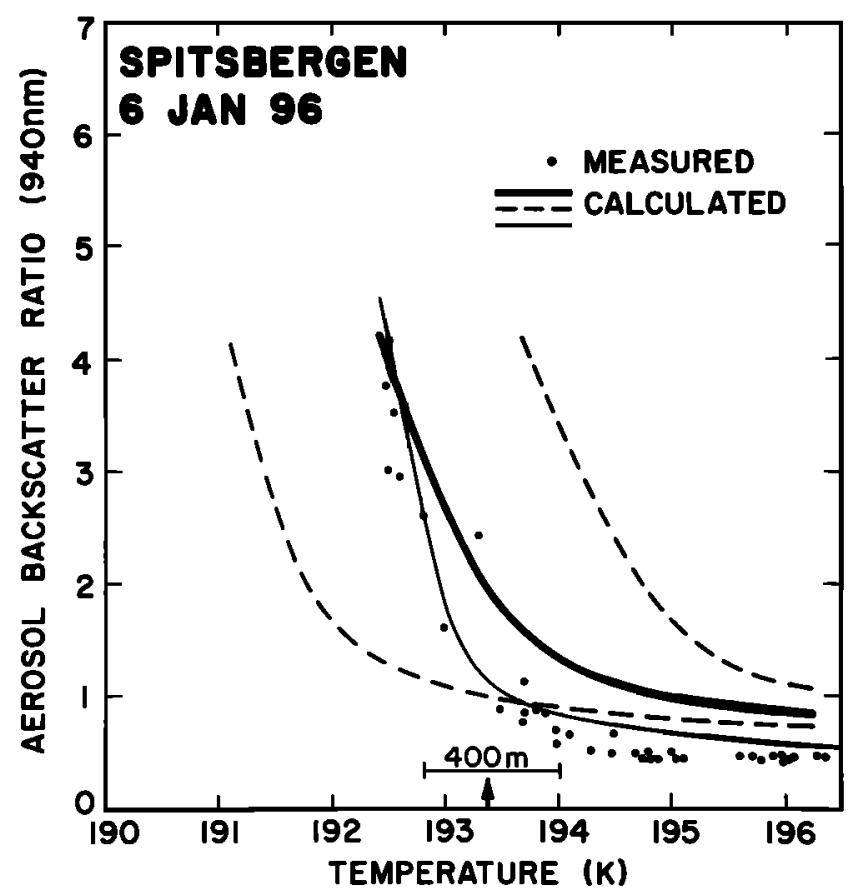

Figure 2. Comparison of measurements made over Spitsbergen on January 6, 1996 near 60-70 mbar with predictions (thick \{thin s solid lines) from the model of Carslaw et al. [1995] using 5\{6\} ppmv water vapor, $7.5\{9\} \mathrm{ppmv}^{\mathrm{HNO}} \mathrm{H}_{3}$, and $0.45\{0.35\}$ ppbv $\mathrm{H}_{2} \mathrm{SO}_{4}$. The dashed lines show the effect of using 6 (right) and 4 (left) ppmv water vapor. The arrow on the temperature scale gives the formally calculated value of $T_{\text {STS }}$ using the thick line and the method described in the text.

reduced for larger values of blue backscatter in the stratosphere. The large variations of color index in the tropospheric clouds are real.

\subsection{Example PSC Threshold}

Figure 2 is an expanded plot of the increase in aerosol backscatter ratio as a function of temperature through the base of the PSC shown in Figure 1 at 60 mbar. The backscatter enhancement occurs over a relatively narrow temperature range. Also shown in the figure are results of calculations (thick solid line) based on the STS model described by Carslaw et al. [1994, 1995] and Luo et al. [1995, 1996] and assuming $5 \pm$ $1 \mathrm{ppmv}$ water vapor, $7.5 \pm 2.5 \mathrm{ppbv} \mathrm{HNO}_{3}$ (consistent with Hopfner et al. [1996]), and $0.45 \pm 0.1 \mathrm{ppbv} \mathrm{H}_{2} \mathrm{SO}_{4}$. Since the range in values of water vapor concentration have the largest influence on the calculation, only the uncertainty associated with this constituent is shown in Figure 2. However, combined uncertainties in the other parameters can noticeably change the slope of the curve and the apparent sharpness of the growth onset region. This effect is illustrated by the thin line which was calculated for 6 ppmv water vapor, $9 \mathrm{ppbv} \mathrm{HNO}_{3}$, and $0.35 \mathrm{ppbv}_{2} \mathrm{SO}_{4}$.

The aerosol backscatter ratio in Figure 2 was calculated from the particle volume (as obtained from the equilibrium STS model) following the ideas and procedure outlined by Hamill et al. [1996]. We have found that this approach yields conversion factors consistent with those previously used in backscattersonde analysis [Rosen et al., 1992, 1993]. The variation in the refractive index of the particles during the initial stages of growth is predicted to be quite minimal [Luo et al.,

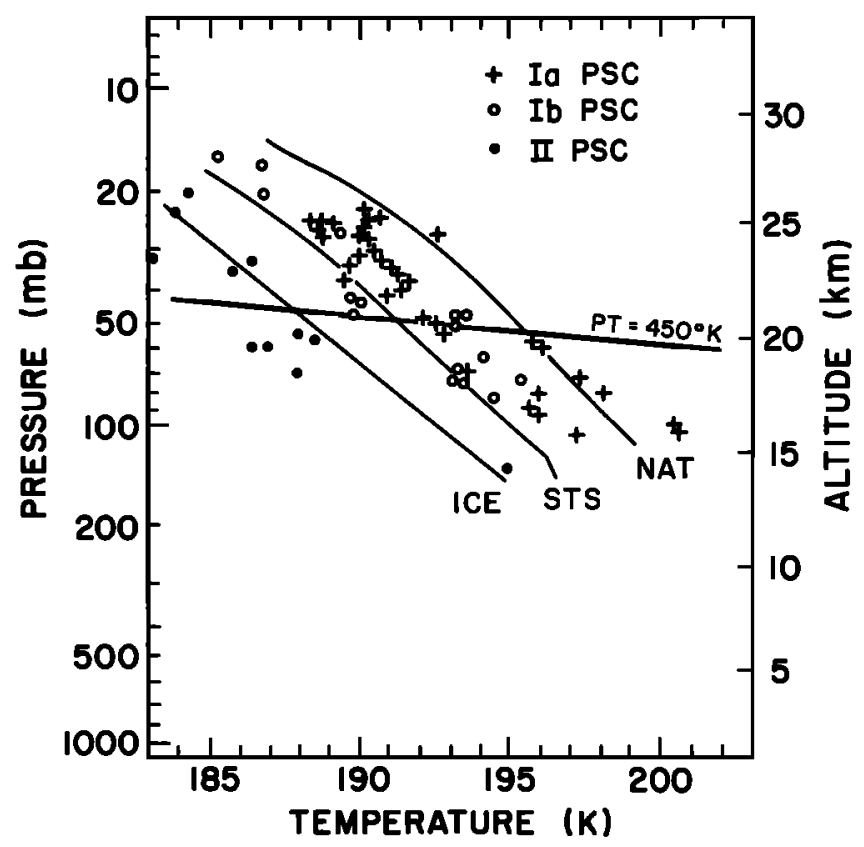

Figure 3. Measured temperatures at locations judged to be the edge of threshold PSC layers. The formation temperatures for ice, supercooled ternary system (STS) PSCs, and nitric acid trihidrate (NAT) PSCs are also shown along with a line of constant potential temperature (PT). The calculations assume 5.0 ppmv $\mathrm{H}_{2} \mathrm{O}, 0.45$ ppbv $\mathrm{H}_{2} \mathrm{SO}_{4}$ and the arctic $\mathrm{HNO}_{3}$ profile given by Hopfner et al. [1996].

1996], and we have used a nominal value of 1.43 . Thus there would appear to be reasonably small uncertainty in the calculation of aerosol backscatter from aerosol volume for the range of particle size distributions expected.

The arrow on the temperature scale in Figure 2 identifies the formally calculated value of the model-derived $T_{\mathrm{STS}}$, which is defined here as the temperature for which the percentage rate of change in the slope of the curve of the particle volume versus temperature is a maximum. For reference in discussions below it should be noted that our definition of $T_{\mathrm{STS}}$ occurs at a temperature where the acrosol backscatter ratio has approximately doubled in the previous $1^{\circ} \mathrm{K}$ temperature drop for the example shown. Thus our $T_{\text {STS }}$ is perhaps as much as $1^{\circ} \mathrm{K}$ below the threshold temperature associated with subjective identification of the PSC edge, or threshold.

\subsection{PSC Thresholds in General}

For the database used here, there are many examples of PSC edge, or threshold, regions such as the one illustrated in Figures 1 and 2 . Figure 3 was constructed in an effort to efficiently summarize the thermodynamic characteristics of these regions. For Figure 3 the threshold point was determined by inspection of sharp cloud edges when the aerosol backscatter ratio began a consistent increase above background. The temperature range for the transition is usually well defined within several tenths of a Kelvin since the signal is increasing rapidly in such a region. The PSC type has been identified according to the scheme discussed below using several distinctive points in, but at the edge of, the cloud.

For reference, various relevant effective saturation vapor pressure curves are also shown in Figure 3. The line labeled ICE is the ice saturation curve for 5 ppmv water vapor. The 


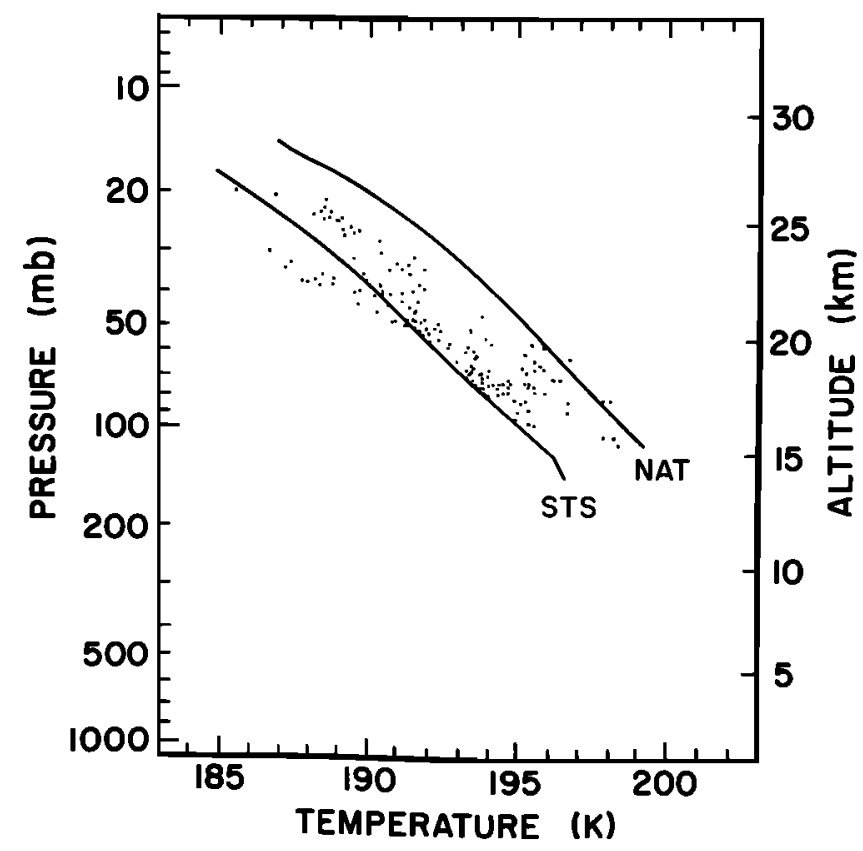

Figure 4. The measured threshold temperatures of PSCs. Each point represents a measurement in which the aerosol backscatter was between $5 \%$ and $20 \%$ above background. The lines marked STS and NAT are the same as in Figure 3.

NAT curve illustrates the expected formation pressure versus temperature of NAT for arctic conditions as taken from Hopfner et al. [1996]. The STS curve was derived using 5 ppmv $\mathrm{H}_{2} \mathrm{O}, 0.45$ ppbv $\mathrm{H}_{2} \mathrm{SO}_{4}$ (consistent with background backscattersonde measurements), and a vertical nitric acid profile consistent with the arctic observations of Hopfner et al. [1996]. Figure 3 would appear to indicate that many of the threshold regions do not show the same good agreement between the calculated $T_{\text {STS }}$ and the observed onset temperature of increased aerosol backscatter as found in Figure 2. Tabazadeh and Toon [1996] have reported similar findings for data related to the Arctic Stratospheric Expedition (ASSE) ER 2 aircraft results. However, within the range of uncertainty in the $T_{\text {STS }}$ calculation it is not clear if the difference between the observed threshold temperature and $T_{\mathrm{STS}}$ is significant. In addition, at least some of the scatter in the points could be attributed to fluctuations in the relevant trace constituent concentrations. Some of the outlying points may be associated with dehydration, which was noted for the 1995-1996 season [Vömel et al., 1997].

Also shown in Figure 3 is an example of a temperaturepressure relationship for a parcel of air undergoing small vertical adiabatic displacements. It is apparent that air mass motions involving even small vertical displacements can lead to temperature excursions covering the entire critical temperature range. Thus possible small-scale vertical motions need to be addressed when deriving a realistic thermal history of PSCs from back-trajectory calculations, as is now well recognized.

\subsection{An Alternate Look at Threshold Temperatures}

It may be noted that the data presented in Figure 3 relates only to well-defined examples of cloud edges and may involve somewhat subjective judgments as to the exact location of the cloud edge. In an effort to eliminate subjective judgments and possibly bias selection all points for which the aerosol back- scatter ratio was between $5 \%$ and $20 \%$ above background have been plotted in Figure 4 for the entire data set. The PSC type has not been shown in this case because it cannot be determined with adequate certainty for some points.

\subsection{Related Issues}

Before further consideration can be given to the interpretation of Figures 3 and 4, other available observational results need to be considered. It would be important to know, for example, if a layer were associated with a cooling or warming air mass. In addition, the results in Figure 3 do not address situations in which PSCs would be expected, but not found, in the observed pressure and temperature environment.

\subsection{Inferring PSC Type From Backscattersonde Data}

The color index has been used to develop an objective method of labeling PSC types using backscattersonde data alone. Figure 5 is a scatterplot illustrating the relation between the aerosol backscatter ratio and the color index for aerosols above the background level in the stratosphere at altitudes $>15 \mathrm{~km}$. The regions of high point population appear to be consistent with the present understanding of the characteristics of PSC types. Figure 6 presents our interpretation and working definition of the aerosol types as derived from the backscattersonde data. The area labeled " $M$ " has a relatively low population and might be tentatively associated with aerosols containing an optically active mixture of both Ia and Ib particles.

The arrow lines in Figure 6 show the calculated evolutionary tracks for several primitive PSC development scenarios initi-

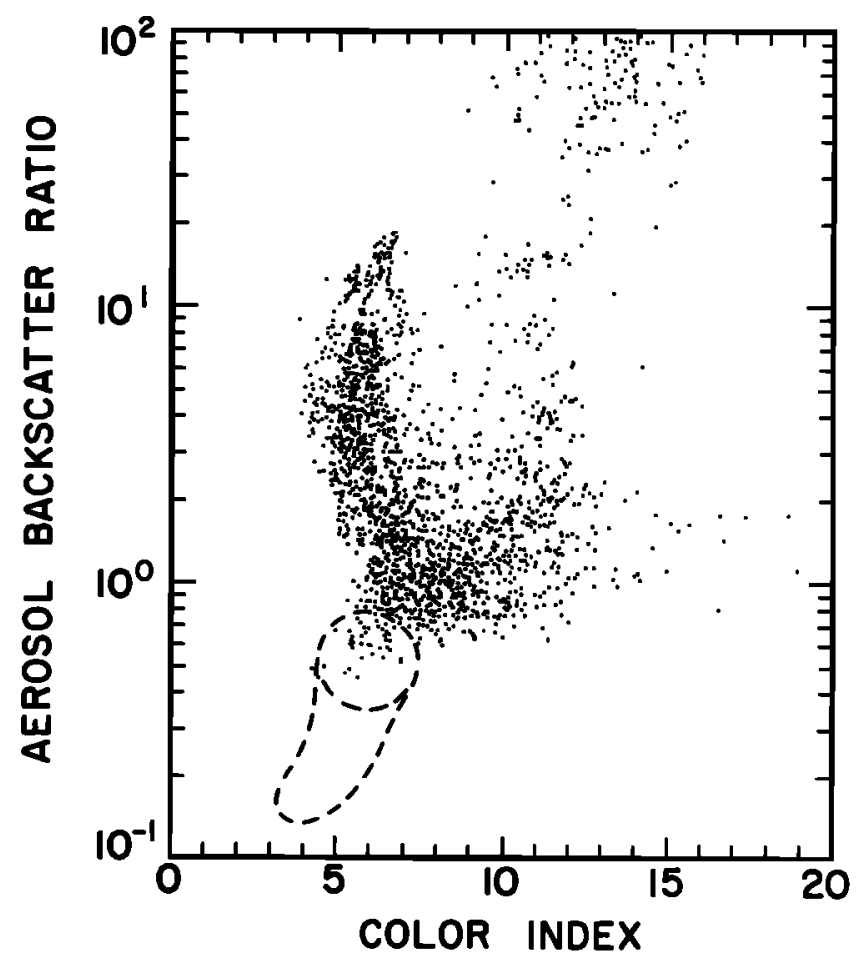

Figure 5. The aerosol backscatter-color index relation for all observed data points with pressures $<110$ mbar and backscatter ratios larger than the background envelope profile given in Figure 1. For reference, points related to the background sulfuric acid aerosol (SAA) fall within the dashed curve and are characterized by a dense clustering within the circular dashed curve. For clarity, this dense cluster of points is not shown. 
ating from the sulfuric acid aerosol (SAA) background with a single-mode log normal size distribution having a starting geometric mean radius of $0.0725 \mu \mathrm{m}$ and a geometric standard deviation $\left(\sigma_{g}\right)$ of 1.86 as suggested by Pinnick et al. [1976]. Scenario 1 relates to all particle growth in which the rate of change in the radius is a constant, which is possible in the low-pressure limit for particles growing at a rate dictated by molecular collisions (simple kinetic theory model). Scenario 2 describes all particle growth in which $\sigma_{g}$ remains constant, as would be expected in the equilibrium STS model as employed by Hamill et al. [1996]. Scenario 3 describes limited participation growth (1 particle in 1000) in which the radius growth rate is a constant for the selected particles and the refractive index is taken to be 1.50 for the growth mode and 1.43 for the background, nongrowth aerosol mode. This scenario may approximately simulate NAT particle formation. Scenario 4 is the same as scenario 3 , except the refractive index is 1.33 for the growth mode and is shown to illustrate the dependency of the results on particle composition. Variations in the index of refraction for scenarios 1 and 2 have relatively small effect on the evolutionary tracks shown in Figure 6.

The four illustrated scenarios in Figure 6 are not meant to be exhaustive. For example, particle evolution from the Ib to Ia region could be simulated. The selected scenarios are presented with the intent of developing an intuitive, probable identification of Ia and Ib PSC regions in the scattering ratio versus color index charts.

The boxed in regions shown in Figure 6 provide a working definition of PSC types based on the backscattersonde observations alone. On the basis of the discussion above the identifications would also appear to be consistent with the previously accepted properties of type Ia and Ib PSCs. These

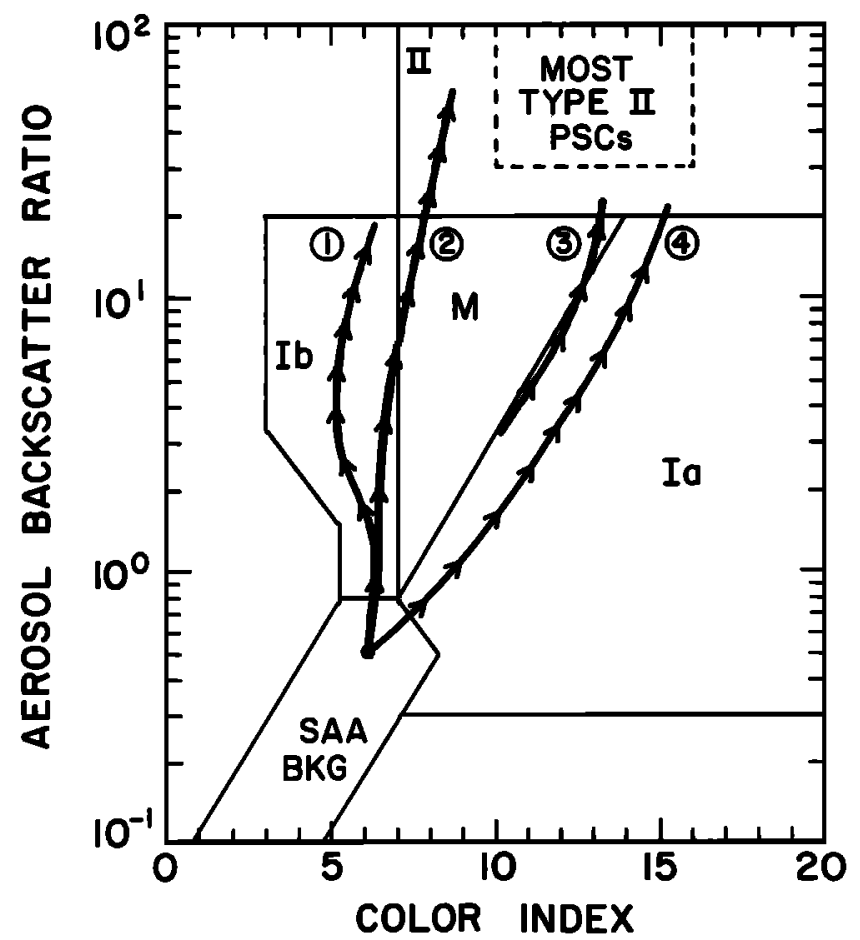

Figure 6. Definition of PSC types using the backscatter-color index relation as obtained with the backscattersonde. The curved lines represent various growth scenarios. See section 6.4 for details.

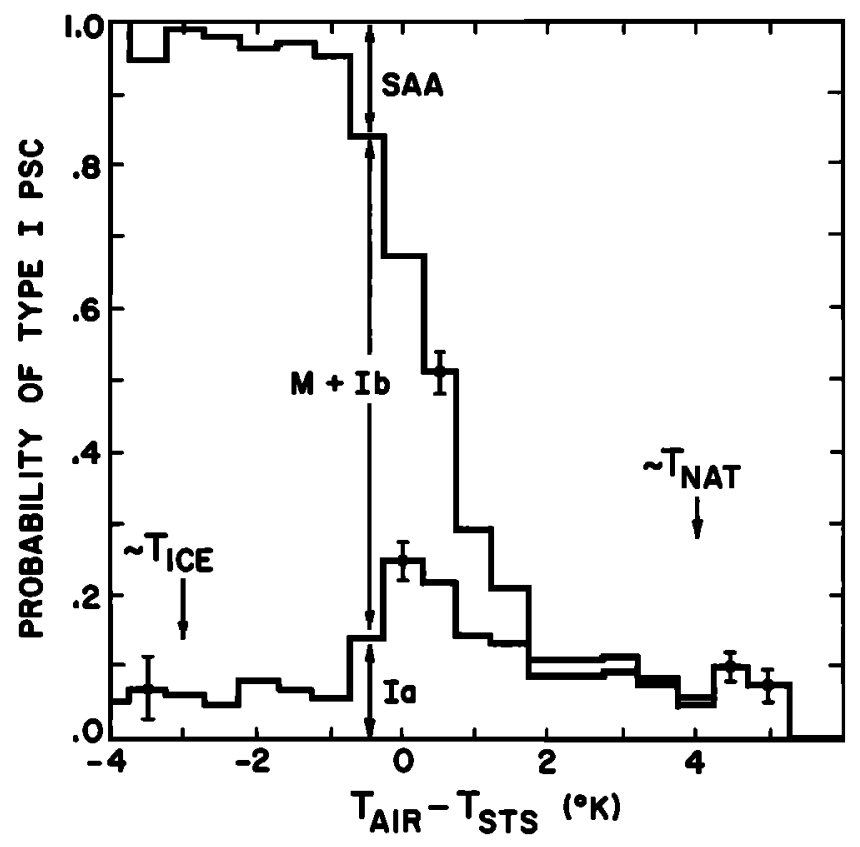

Figure 7. The probability of observing a type I PSC as a function of temperature. Error bars represent \pm 1 standard deviation as computed from the available number of data points. The arrows under $T_{\text {ice }}$ and $T_{\text {NAT }}$ indicate the approximate values for these two parameters for the ambient conditions and assumptions described in the text. The arrow lengths associated with SAA, M + Ib, and Ia illustrate their respective probability of occurrence.

definitions provide an objective approach to investigating systematic differences in threshold temperatures for various PSC types.

\subsection{Probability of Type I PSCs}

As mentioned above, important information is missing from Figure 3 in that it may be necessary to consider all of the observations, particularly those for which PSCs were not detected but were expected in the observed pressure-temperature regime. Figure 7 was constructed from all of the data points above $15 \mathrm{~km}$ and utilized the working definition of the PSC types described above. As will be discussed below, these results present a distinctly different impression than might be gained from Figure 3 alone.

\subsection{Probability of Type II PSCs}

Figure 8 is similar in nature to Figure 7 but is only related to type II PSC occurrence. In a sense, Figure 8 represents a control experiment in that a dramatic increase in type II PSC observations near the ambient temperature of $T_{\text {ice }}$ is expected. Experimentally, there is, in fact, a large increase in type II PSC probability (going from $\sim 0.05$ to $\sim 0.95$ ) within $\sim \pm 1 \mathrm{~K}$ of $T_{\text {ice }}$. This result lends credibility and significance to the analysis given in Figure 7 and provides an independent assessment of the probable temperature accuracy, resolution, and data noise associated with the results.

\section{Discussion}

\subsection{Relation to Other Backscattersonde Studies}

An in-depth investigation of the temperature histories of well-developed PSC layers observed with the backscattersonde 


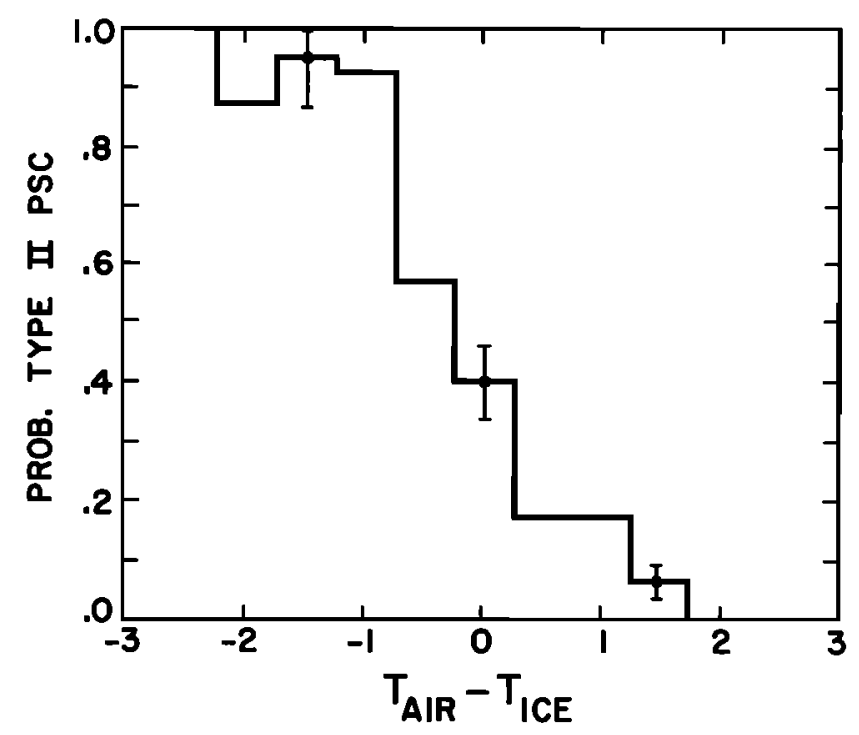

Figure 8. The probability of observing a type II PSC as a function of temperature.

has recently been given by Larsen et al. [1994, 1995, 1996, 1997]. These results show that type Ib PSCs tend to be observed in newly formed clouds or shortly after entering a cold region. The temperature histories of type Ia PSCs typically involve an extended cold period at temperatures below $T_{\text {NAT }}$ for one or more days prior to observation. Tabazadeh et al. [1996] have also concluded that exposure to temperatures $<T_{\text {NAT }}$ for approximately a day or more should be the main criteria for type Ia occurrence. For reference, below the reader is reminded that the PSCs considered in Figure 3 are at the threshold level, while the PSCs studied by Larsen et al. are significantly above threshold scattering values and thus may display somewhat different properties.

\subsection{Air Mass Trajectories}

Air mass back trajectories have been calculated for the examples of PSC threshold regions represented in Figure 3. In almost all cases the synoptic air temperature was relatively steady or decreasing. A few notable exceptions in which the air temperature was increasing are associated with points near and above the NAT curve. Unfortunately, there are not enough warming examples to be decisive. In addition, as discussed below, mesoscale temperature variations could impose significant short-term temperature variations that may, but not necessarily, override the results deduced from a synoptic analysis. Larsen et al. [1997] present convincing evidence that synoptic temperature variations can, in fact, still play an important role in the formation and evolution of PSC types.

\subsection{Formation Time Constants}

In considering the significance of PSC threshold conditions it is important to consider the characteristic time that the particles need to grow from the initial background size to a state that can be distinctly recognized by the observational technique. Meilinger et al. [1995] have modeled the growth of background size aerosols for the STS system under fast cooling conditions. Inspection of their results indicates that $0.10 \mu \mathrm{m}$ particles can increase by more than $10 \%$ in size per $10 \mathrm{~min}$ in the region of growth. Estimates given by Murphy and Gary [1995] suggest a 7\% change in radius in $10 \mathrm{~min}$. The time required for the aerosols to grow to the threshold detection level of the backscattersonde would be smaller than this estimate, probably only a few minutes. It is apparent that threshold PSCs have the potential of responding quickly to their environmental changes and thus to the mesoscale temperature fluctuations. The timescale for mesoscale fluctuations is of the order of 10 hours for the conditions described by Murphy and Gary [1995] and may be $\sim 10 \mathrm{~min}$ for wave clouds.

For a simple kinetic theory model, particle growth and evaporation rates are essentially the same for the background size mode involved here, although the detailed model of Meilinger et al. [1995] shows some noticeable hysteresis effects for a growth-evaporation cycle. If there is a nucleation barrier involving the onset of growth, the particle may be expected to evaporate at a higher temperature than that of formation. In addition, small accommodation (sticking) coefficients could lead to long formation times but could maintain fast evaporation. Such possibilities may be useful for interpreting some of our observations, particularly those characteristic of Ia PSCs.

As indicated above, it is possible to imagine conditions under which the growth rate could be much smaller, as, for example, in the case of aerosol particles that have very limited surface sites on which condensation or growth can occur or in which the effective accommodation coefficient is very small. In this situation the particles may need to be at temperatures below the effective saturation point for a long time before they reach the detection threshold, but they could evaporate quickly at temperatures just above the saturation point. Mesoscale temperature fluctuations would prevent easy detection of such particles near the saturation or growth point because they would go through cycles of slow growth and fast evaporation. It might be expected that such particles could be easily detected only in air masses that have spent adequate time at temperatures sufficiently below the saturation point, where mesoscale temperature fluctuations do not regularly bring the air mass above the evaporation point. Such characteristics would seem to describe some of the known properties of Ia PSCs as discussed by Larsen et al. [1997].

\subsection{Color Index Distinctions of PSCs}

Intuitively, one might expect to be able to identify two general groupings of data points in Figure 3 according to their association with type Ia or Ib PSCs. However, within the main body of points, there does not seem to be a distinct difference between various type I PSCs. On the other hand the outlying points tend to be associated with $T_{\mathrm{STS}}$ for type Ib PSCs and with $T_{\mathrm{NAT}}$ for type Ia PSCs. A possible reason that the warm outlying points are associated with $T_{\mathrm{NAT}}$ is that this reflects the approximately correct evaporation temperature of type Ia PSCs. Type Ib PSCs are not expected to be present for the same conditions because they would have evaporated at significantly lower temperatures.

The failure of Figure 3 to clearly distinguish between Ia and Ib PSCs may be related to the idea that NAT PSCs cannot be readily detected until the temperature has been below $T_{\text {NAT }}$ for some time, and this lower temperature regime is, by chance, also close (within natural variations) to the effective formation temperature of type Ib (STS) PSCs. The more difficult question of which type of PSC (Ia or $\mathrm{Ib}$ ) is predicted to form has been addressed by Larsen et al. [1997]. 


\subsection{Probability of PSC Occurrence}

Figure 7 shows a dramatic increase in the occurrence probability of $\mathrm{M}+\mathrm{Ib}$ PSCs near $T_{\mathrm{STS}}$, as would be expected for an aerosol system with a distinct temperature range of condensation. The probability of type Ia occurrence is consistent with a constant value of roughly $10 \%$ at temperatures below $T_{\text {NAT }}$. The variations in this probability could be due to errors in the identification of PSC types and may not be of any real significance. Thus variations or structure in the Ia occurrence curve may not be reproducible from year to year. In contrast, the $\mathrm{M}+\mathrm{Ib}$ occurrence curve is expected to be reproducible for STS aerosols.

\subsection{Type Ic PSC Considerations}

Figures 5 and 6 show a number of observations occurring in the mixed mode $(\mathrm{M})$ region with aerosol scattering ratios and color indexes near 10. Optical model calculations indicate that these particles would contain considerably more nitric acid than is available to be consistent with either NAT or STS PSCs. Thus these particles appear to contain an excess amount of water and are perhaps associated with pre-type II PSCs, even though their backscatter is much too small to be classified as an ordinary type II PSC. Their water-rich composition invites speculation that they may be associated with the type Ic PSCs proposed by Tabazadeh and Toon [1996], although it is not clear that they meet the color index criteria.

\subsection{Deliquescence Temperature Issues}

Koop and Carslaw [1996] have described a deliquescence temperature $T_{d}$ which represents the melting and sudden onset of growth for cooling, frozen sulfuric acid tetrahydrate (SAT) particles. Using our definition of $T_{\mathrm{STS}}$, we find that $T_{d}$ and $T_{\text {STs }}$ are essentially identical for practical purposes. Thus our threshold temperatures themselves cannot be used to identify initially frozen or liquid particles. However, in the frozen particle deliquescence case the growth starts suddenly at $T_{d}$, while for the liquid STS system, there is a small but significant growth or particle swelling (deliquescence) before $T_{\mathrm{STS}}$ is reached. In Figure 2 the liquid deliquescence swelling effect can be noted in the difference between the solid line and the data points: at much warmer temperatures $\left(215^{\circ} \mathrm{K}\right)$ the thick solid line would approach the horizontal line defined by the data points shown at temperatures $>195^{\circ} \mathrm{K}$.

A comparison of the measured data points with the thick line theoretical curve in Figure 2 might suggest that the growth onset is more abrupt than would be expected for STS aerosols. However, within the range of parameter uncertainty (thin line prediction) the onset could be much sharper and probably indistinguishable from the sudden onset that would be expected in the deliquescence model. Thus it is not clear to us that our observed sharp PSC onsets are necessarily indicative of solid (frozen) particle deliquescence.

\subsection{Type Ib Growth Models}

The type Ib PSC growth scenarios illustrated in Figure 6 suggest that a better agreement is obtained with the measured values in Figure 5 for a model in which the width of the size distribution narrows with increasing size or backscatter. The STS model employed by Meilinger et al. [1995], which is applied to relatively fast cooling conditions, shows a significant narrowing of the size distribution as growth occurs. On the other hand, the STS model described by Hamill et al. [1996] is consistent with a constant size distribution width if the initial and final $\mathrm{H}_{2} \mathrm{SO}_{4}$ weight fractions of the droplets are not a function of particle size.

\subsection{Relation to Satellite Observations}

Satellite observations of PSCs have been used to generate a figure by Poole and Pitts [1994] similar to our Figures 3 and 4. Their winter arctic results for a 0.5 probability of observing a PSC as a function of altitude is very closely approximated by the line labeled NAT in our Figures 3 and 4, suggesting that type Ia PSCs were dominant and generally present near $T_{\text {NAT }}$. On first consideration this result may seem inconsistent with the backscattersonde observations because our results show that the PSC threshold temperature is systematically below $T_{\text {NAT }}$ and that the probability of observing a PSC is greatly enhanced near $T_{\mathrm{STS}}$, not $T_{\mathrm{NAT}}$. However, a more in-depth understanding of the two data sets indicates that they are probably entirely in agreement and provide some new insight. The satellite measurements sample conditions over a long horizontal path and could have a high probability of registering a PSC even if the PSC coverage were relatively small. Figure 7 indicates that one might expect about a $10 \%$ PSC coverage for stratospheric temperatures near and just below $T_{\text {NAT }}$. Thus a balloon sounding would have only a small chance of going through a type Ia (NAT) PSC at these temperatures, while the satellite could have a high chance of detecting a NAT PSC somewhere in the long horizontal observing path. Furthermore, this interpretation suggests that it may be difficult, or perhaps impossible, to detect PSC enhancements at $T_{\text {STS }}$ temperatures through satellite-based observations because the phenomena could be masked by localized sparse type Ia PSC activity.

\section{Conclusions}

Aerosol backscatter and concurrent temperature variations near the edge of some PSCs are consistent with the known optical properties of type Ib PSCs and the thermodynamic model of STS aerosols as proposed by other investigators. However, there appears to be about a $1^{\circ} \mathrm{K}$ offset in the predicted and observed average threshold temperatures, which may not be significant in light of experimental errors, accuracy of the technique, and assumptions of trace gas components. In addition, the observed threshold temperatures range between $T_{\text {NAT }}$ and our defined $T_{\text {STS }}$ (which is also close to $T_{d}$ ).

The available data do not show an easily distinguishable difference between the threshold temperatures for type Ia and Ib PSCs. In contrast, the probability of occurrence curves for type Ia and Ib are quite dissimilar in that the Ib PSCs show a dramatic increase near $T_{\text {STS }}$ (as expected from present theories) and the type Ia PSCs display a relatively low, but very roughly uniform, probability at all measured temperatures below about $T_{\text {NAT }}$. The probability of occurrence for Ia PSCs is consistent with select nucleation and slow particle growth at temperatures below about $T_{\text {NAT }}$ and relatively rapid evaporation near $T_{\text {NAT }}$.

Acknowledgments. This work has been supported by the National Science Foundation under a grant from the Office of Polar Programs and in cooperation with a supporting effort by the Commission of the European Union, the Danish Space Board, and the Commission for Scientific Research in Greenland.

\section{References}

Beyerle, G., B. Luo, R. Neuber, T. Peter, and I. S. McDermid, Temperature dependence of ternary solution particle volumes as ob- 
served by lidar in the Arctic stratosphere during winter 1992/1993, J. Geophys. Res., 102, 3603-3609, 1997.

Browell, E. V., C. F. Butler, S. Ismail, P. A. Robinette, A. F. Carter, N. S. Higdon, O. B. Toon, M. R. Schoeberl, and A. F. Tuck, Airborne lidar observations in the wintertime Arctic stratosphere: Polar stratospheric clouds, Geophys. Res. Lett., 17, 385-388, 1990.

Carslaw, K. S., B. P. Luo, S. L. Clegg, T. Peter, P. Brimblecombe, and P. J. Crutzen, Stratospheric aerosol growth and $\mathrm{HNO}_{3}$ gas phase depletion from coupled $\mathrm{HNO}_{3}$ and water uptake by liquid particles, Geophys. Res. Lett., 21, 2479-2482, 1994.

Carslaw, K. S., B. P. Luo, and T. Peter, An analytic expression for the composition of aqueous $\mathrm{HNO}_{3}-\mathrm{H}_{2} \mathrm{SO}_{4}$ stratospheric aerosols including gas phase removal of $\mathrm{HNO}_{3}$, Geophys. Res. Lett., 22, 1877$1880,1995$.

Crutzen, P. J., and F. Arnold, Nitric acid cloud formation in the cold Antarctic stratosphere: A major cause for the springtime "ozone hole," Nature, 324, 651-655, 1986.

Drdla, K., A. Tabazadeh, R. P. Turco, M. Z. Jacobson, J. E. Dye, C. Twohy, and D. Baumgardner, Analysis of the physical state of one Arctic polar stratospheric cloud based on observations, Geophys. Res. Lett., 21, 2475-2478, 1994.

Dye, J. E., et al., In situ observations of an Antarctic polar stratospheric cloud: Similarities with Arctic observations, Geophys. Res. Lett., 23, 1913-1916, 1996.

Fahey, D. W., K. K. Kelly, G. V. Ferry, L. R. Pool, J. C. Wilson, D. M. Murphy, M. Loewenstein, and K. R. Chan, In situ measurements of total reactive nitrogen, total water, and aerosol in a polar stratospheric cloud in the Antarctic, J. Geophys. Res., 94, 11,299-11,315, 1989

Hamill, P., A. Tabazadeh, S. Kinne, O. B. Toon, and R. P. Turco, On the growth of ternary system $\mathrm{HNO}_{3} / \mathrm{H}_{2} \mathrm{SO}_{4} / \mathrm{H}_{2} \mathrm{O}$ aerosol particles in the stratosphere, Geophys. Res. Lett., 23, 753-756, 1996.

Hanson, D. R., and K. Mauersberger, Laboratory studies of nitric acid trihydrate: Implications for the south polar stratosphere, Geophys. Res. Lett., 15, 855-858, 1988.

Hopfner, M., C. E. Blum, T. Blumenstock, H. Fischer, and T. Guide, Evidence for the removal of gaseous $\mathrm{HNO}_{3}$ inside the Arctic polar vortex in January 1992, Geophys. Res. Lett., 23, 149-152, 1996.

Koop, T., and K. S. Carslaw, Melting of $\mathrm{H}_{2} \mathrm{SO}_{4} \cdot 4 \mathrm{H}_{2} \mathrm{O}$ particles upon cooling: Implications for polar stratospheric clouds, Sczence, 272, 1638-1641, 1996.

Larsen, N., B. Knudsen, T. S. Jorgensen, A. di Sarra, D. Fua, P. Di Giroloamo, G. Flocco, M. Cacciani, J. M. Rosen, and N. T. Kjome, Backscatter measurements of stratospheric aerosols at Thule during January-February 1992, Geophys. Res. Lett., 21, 1303-1306, 1994.

Larsen, N., J. M. Rosen, N. T. Kjome, and B. Knudsen, Deliquescence and freezing of stratospheric aerosol observed by balloon-borne backscattersondes, Geophys. Res. Lett., 22, 1233-1236, 1995.

Larsen, N., B. M. Knudsen, J. M. Rosen, N. T. Kjome, and E. Kyro, Balloon-borne backscatter observations of type 1 PSC formation: Inference about physical state from trajectory analysis, Geophys. Res. Lett., 23, 1091-1094, 1996.

Larsen, N., B. M. Knudsen, J. M. Rosen, N. T. Kjome, R. Neuber, and E. Kyrö, Temperature histories in liquid and solid PSC formation, $J$. Geophys. Res., 102, 23,505-23,517, 1997.

Luo, B. P., K. S. Carslaw, T. Peter, and S. L. Clegg, Vapor pressures of $\mathrm{H}_{2} \mathrm{SO}_{4} / \mathrm{HNO}_{3} / \mathrm{HCl} / \mathrm{HBr} / \mathrm{H}_{2} \mathrm{O}$ solutions to low stratospheric temperatures, Geophys. Res. Lett., 22, 247-250, 1995.

Luo, B., U. K. Krieger, and T. Peter, Densities and refractive indices of $\mathrm{H}_{2} \mathrm{SO}_{4} / \mathrm{HNO}_{3} / \mathrm{H}_{2} \mathrm{O}$ solutions to stratospheric temperatures, Geophys. Res. Lett., 23, 3707-3710, 1996.

Manney, G. L., M. L. Santee, L. Froidevaux, J. W. Waters, and R. W. Zurek, Polar vortex conditions during the 1995-1996 Arctic winter: Meteorology and MLS ozone, Geophys. Res. Lett., 23, 3203-3206, 1996.

Meilinger, S. K., T. Koop, B. P. Luo, T. Huthwelker, K. S. Carslaw, U. Krieger, P. J. Crutzen, and T. Peter, Size-dependent stratospheric droplet composition in lee wave temperature fluctuations and their potential role in PSC freezing, Geophys. Res. Lett., 22, 3031-3034, 1995.

Murphy, D. M., and B. L. Gary, Mesoscale temperature fluctuation and polar stratospheric clouds, J. Atmos. Sci., 52, 1753-1760, 1995.

Naujokat, B., and S. Pawson, The cold stratospheric winters 1994/1995 and 1995/1996, Geophys. Res. Lett., 23, 3703-3706, 1996.

Pinnick, R. G., J. M. Rosen, and D. J. Hofmann, Stratospheric aerosol measurements, III, Optical model calculations, J. Atmos. Sci., 33, 304-314, 1976.

Poole, L. R., and M. C. Pitts, Polar stratospheric cloud climatology based on stratospheric aerosol measurement, II, Observations from 1978 to 1989, J. Geophys. Res., 99, 13,083-13,089, 1994.

Rosen, J. M., and N. T. Kjome, The backscattersonde: A new instrument for atmospheric aerosol research, Appl. Opt., 30, 1552-1561, 1991.

Rosen, J. M., N. T. Kjome, and H. Fast, Penetration of Mount Pinatubo aerosols into the north polar vortex, Geophys. Res. Lett., 19, 1751-1754, 1992.

Rosen, J. M., N. T. Kjome, and S. J. Oltmans, Simultaneous ozone and polar stratospheric cloud observations at south pole station during winter and spring 1991, J. Geophys. Res., 98, 12,741-12,751, 1993.

Santee, M. L., G. L. Manney, W. G. Read, L. Froidevaux, and J. W. Waters, Polar vortex conditions during the 1995-1996 Arctic winter: MLS CLO and $\mathrm{HNO}_{3}$, Geophys. Res. Lett., 23, 3207-3210, 1996.

Schoeberl, M. R., and P. A. Newman, A multiple level trajectory analysis of vortex filaments, J. Geophys. Res., 100, 25,801-25,815, 1995.

Tabazadeh, A., and O. B. Toon, The presence of metastable $\mathrm{HNO}_{3}$ $\mathrm{H}_{2} \mathrm{O}$ solid phases in the stratosphere inferred from ER 2 data, $J$. Geophys. Res., 101, 9071-9078, 1996.

Tabazadeh, A., R. P. Turco, K. Drdla, M. Z. Jacobson, and O. B. Toon, A study of type I polar stratospheric cloud formation, Geophys. Res. Lett., 21, 1619-1622, 1994a.

Tabazadeh, A., R. P. Turco, and M. Z. Jacobson, A model for studying the composition and chemical effects of stratospheric aerosols, $J$. Geophys. Res., 99, 12,897-12,914, 1994b.

Tabazadeh, A., O. B. Toon, B. L. Gary, J. T. Bacmeister, and M. R. Schoeberl, Observational constraints on the formation of type Ia polar stratospheric clouds, Geophys. Res. Lett., 23, 2109-2112, 1996.

Taleb, D. E., J. L. Ponche, and P. Mirabel, Vapor pressures in the ternary system water-nitric acid-sulfuric acid at low temperaturc: A reexamination, J. Geophys. Res., 101, 25,967-25,977, 1996.

Toon, O. B., P. Hamill, R. P. Turco, and J. Pinto, Condensation of $\mathrm{HNO}_{3}$ and $\mathrm{HCl}$ in the winter polar stratosphere, Geophys. Res. Lett., $13,1284-1287,1986$

Toon, O. B., E. V. Browell, S. Kinne, and J. Jordan, An analysis of lidar observations of polar stratospheric clouds, Geophys. Res. Lett., 17, 393-396, 1990.

Vömel, H., M. Rummukainen, R. Kivi, J. Karhu, T. Turunen, E. Kyro, J. Rosen, N. Kjome, and S. Oltmans, Dehydration and sedimentation of ice particles in the Arctic stratospheric vortex, Geophys. Res. Lett., 24, 795-798, 1997.

I. Beninga and R. Neuber, Alfred Wegner Institute of Polar and Marine Research, Research Unit Potsdam, D-14473 Potsdam, Germany.

J. Karhu, R. Kivi, and E. Kyrö, Finnish Meteorological Institute, Sodankylä Observatory, SF99600 Sodankylä, Finland.

N. T. Kjome and J. M. Rosen, Department of Physics and Astronomy, University of Wyoming, Laramie, WY 82071. (wyojim@igc.apc. org)

B. M. Knudsen and N. Larsen, Danish Meteorological Institute, Lyngbyvej 100, DK-2100 Copenhagen, Denmark.

(Received June 25, 1997; revised September 16, 1997; accepted September 19, 1997.) 